\title{
An Instrumented Insole System for Gait Monitoring and Analysis
}

\author{
http://dx.doi.org/10.3991/ijoe.v10i6.3971 \\ Anas M.N. \\ Universiti Malaysia Perlis, Pauh, Arau, Malaysia
}

\begin{abstract}
Insole pressure during walking or running could be used to analyze abnormality of kinetic measurement for broad applications as such a diabetic foot, knee injuries and neural disorder. Moreover, some application of biometrics and human behavior also had been identified based on gait pattern. This project purposed a prototype for measuring ground reaction force using developed an instrumented insole measurement system based on dual-parameters of gait monitoring and analysis; foot force and plantar flexion. The system comprised hardware such as force and angle sensors, signal conditioning circuit and data acquisition device and software for signal's visualization for user interface and real-time gait analysis. The sensors will be placed on top of the shoe insole for measuring force distribution on foot and foot plantar for measuring flexion angle wired to a data acquisition device. The locations of the sensors have been determined based on the footprint during the gait cycle. This paper also presented details of components involved in the measurement system. It has been demonstrated that the developed system prototype could be used in the clinical gait monitoring and analysis established based on the foot force and plantar angle starting from heals strike till toe-off positions.
\end{abstract}

Index Terms-Foot force, gait monitoring, instrumented insole, real-time gait analysis.

\section{INTRODUCTION}

In this sections, some literature on need of gait analysis, methods used in the analysis, the gait cycle and related prior studies is discuss to bring up the understanding of the research needs.

\section{A. The needs of gait analysis}

Gait analysis is a study of human walking, using the eye and brain of experienced observers, augmented by instrumentation for measuring body mechanics, kinetics and muscle movement. There are many factors affecting the gait pattern such as physicals, physiological, pathological and sometimes influence by emotions. However, the gait pattern could be either a temporary or permanent [1]. It has been claimed by many researchers that gait pattern could provide information on health conditions and few diseases such as knee osteoarthritis, diabetic foot, Parkinson is affected and observed from gait due to its unique fingerprint, which sometimes resulted antalgic, apraxic and ataxic pattern [2-5].Furthermore, gait analysis also could be used to make detailed diagnosis, prognosis for certain diseases and also in the rehabilitation evaluation and treatment [6].

\section{B. Types and techniques of gait analysis}

There are two types of gait analysis, manual observations by experienced physicians to monitor and observed gait abnormality and secondly by using advanced computer-aided tools to analyze the gait pattern [7]. The gait analysis normally analyzed few parameters such as step length, stride length, cadence, speed, foot angle and much more $[8,9]$. In the first method of gait observation, it is required a highly trained expert to interpret the gait abnormality which sometimes caused a higher chance resulted an inconsistency analysis compared to the computerbased system. This is because it needs a precision observation of the gait features are assessed and analyzed qualitatively during a visual examination [10]. Meanwhile, the computer-aided system is most popular, which rely on the computer system which is highly complex, costly instrument and also need a trained person to analyze the gait pattern. However, this system is more reliable, repeatable and high-accuracy measurement employing hightechnology sensors and transducers.

\section{The gait cycles}

The gait cycle includes both the complex body appearance and dynamic of human walking motion [11]. The two basic components in the gait cycle are, the swing phase where the foot is in the air for limb advancement, and the stance phase where the limb is contact to the ground [12]. Gait pattern of each individual is varied and the element performing gaits usually change over time. As discussed earlier, many factors need to be considered in analyzing a gait pattern. The gait cycle starting from heel strike, foot flat, heel off, toe-off and back to heel strike again for a complete cycle. For each step, time defines the time between one foot hitting the floor and the other foot hitting the floor [13]. Each person has a unique time period and distributed foot regions pressure according to physical parameters such as body balance, weight, height, age, motor function and foot pressure sense [14]. However, this unique pattern could be differentiating between normal and abnormal gait pattern. According to force distribution on the feet, a person with normal gait will show a result with a symmetrical distribution of a force compared with the abnormal gait pattern $[15,16]$.

\section{METHODOLOGY}

The hardware compromised in this system is developed based on the literature review which suggesting certain important aspect such as sensor selection, signal conversion and acquisition and suitable locations of sensor placement on the instrumented insole in order to get a reliable analysis for measurement of foot force and plantar flexion angle. In the software section, sensor's non-linear 
response and user interface are developed to ensure reliability of the measurement system and also providing a real-time analysis. Procedures of experiment also are presented in this section.

\section{A. Sensors}

Force sensing sensors (FSR) model 402 (from Interlink electronic) and flexible bend sensor (FBS) model FLX-01 (from Spectra Symbol) is used in this research. These sensors are resistive based sensors which, the electrical resistance changes under tension or compression of the sensor. The active area of FSR sensors is $12.7 \mathrm{~mm}$ diameter and $95.25 \mathrm{~mm}$ of active length for FBS sensor. The FSR and FBS are very popular due to its cost-effective and suitable for measuring foot force and flexion. The FSR and FBS sensor are based on the polymer thick film (PTF) material, which exhibits a decrease in resistance when increase the force applied at the surface for FSR and increase of resistance when increasing of bend angle for FBS. Both sensor's accuracy determining force and bend angle ranges from $\pm 5 \%$ to $\pm 25 \%$ in $10 \mathrm{~K}$ ohms to $100 \mathrm{~K}$ ohms for FSR and $\pm 30 \%$ from $60 \mathrm{Kohms}$ to $110 \mathrm{~K}$ ohms for FBS sensor [21]. These sensors are not a high accuracy or precision, resolution sensors. However, in this research application, the sensor accuracy and repeatability in measurement could be improved by help of developed software. Figure 1 show the four FSR sensors are placed in the shoe insole to the selected foot regions, and FBS sensor is placed at the bottom of the insole for measuring plantar flexion.

\section{B. Electronic Circuitry and Data Acquisition}

Sensors are connected to the signal conditioning circuit. This circuit consists of four input and four output of a single operational amplification (Op-amp) device circuit, which convert the changing of resistant (sensor output) will change the op-amp output voltage. Op-amp model LM324AN from STMicroelecronic used a voltage divider configuration. Equation (1) shows output voltage across the conditioning circuit for both FSR and FBS sensor.

$$
\text { Vout }=\frac{V x}{\left(1+\frac{R z}{R 1}\right)}
$$

Where, $\mathrm{Vx}$ voltage applied to the divider circuit, $\mathrm{RZ}$ is the resistance value from FSR or FBS sensor, and R1 is fixed circuit divider resistance. The USB data acquisition device (DAQ) model 6210 from National Instrument is used in this project. Five analog voltage output form signals conditioning circuit are connected across the analog input to the DAQ device. This device is set to accomplish sampling signals for $10 \mathrm{~Hz}$ sample per second $(\mathrm{S} / \mathrm{s})$ and connected to the laptop over a USB connection for further signal processing.

\section{Software}

LabVIEW graphical language software from National Instrument use in this system is uncomplicated to be implemented compared to other's software platform, which depends on descriptive programming. The main process program consists of the sensor's calibration algorithm identified earlier for each sensor and also sensors signal's visualization window for analysis and data logging. In order to improved accuracy and linearity of the sensor's output characteristics and measurement system, a sensor calibration is needed. In this study, a static calibration using is performed to obtain the relationship between the applied force and the output voltage. The sensors are calibrated using Instron 5848 test machine and also LabVIEW software method by applying the sensor response curve using 3rd order polynomial regression model is obtained. Equation (2) shows the FSR conversion from voltage to force (Newton) for FSR sensors.

$$
y=-A+A x-A x^{2}+A x^{3}
$$

Where, $\mathrm{y}$ is force (Newton), $\mathrm{A}$ is a numeric value and $\mathrm{x}$ is weight $(\mathrm{kg})$. This equation determines the best fit for all four FSR sensors used in this system. For FBS sensor, voltage output also been calibrated using a regression model to convert from voltage value to angle in order to measure the plantar flexion of the foot. Equation (3) shows the regression model.

$$
y=A-A x
$$

Where $\mathrm{y}$ is the output in degree, $\mathrm{A}$ is a numeric value and $\mathrm{x}$ is the voltage measured from FBS sensor.

Another software use in this system is Kinovea software. Kinovea is a video analysis software use for analysis human kinetics in many applications. This software will help to determine the angle of plantar flexion during experiment could and also compared to the actual of FBS output value. Figure 2 shows the LabVIEW front panel display. For FSR sensors, Y-axis will show "Force" value in Newton and for FBS sensor, Y-axis shows the plantar flexion angle.

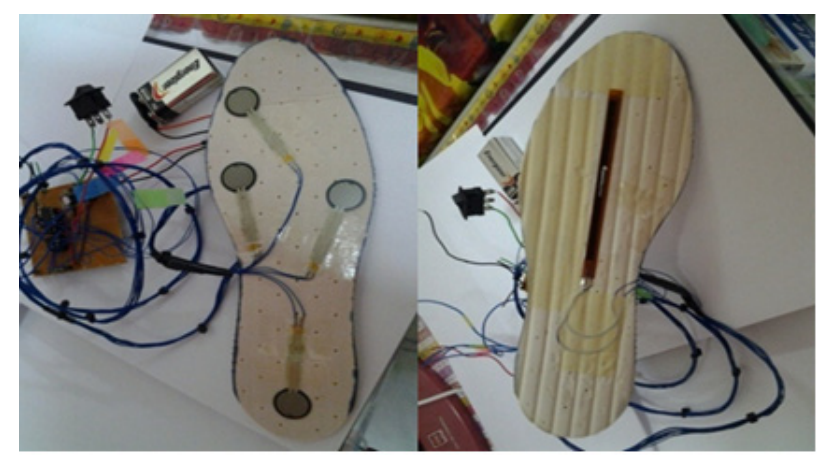

Figure 1. FSR and FBS sensor's position on the insole. The FSR placement is on top of the insole, and FBS is below the insole.

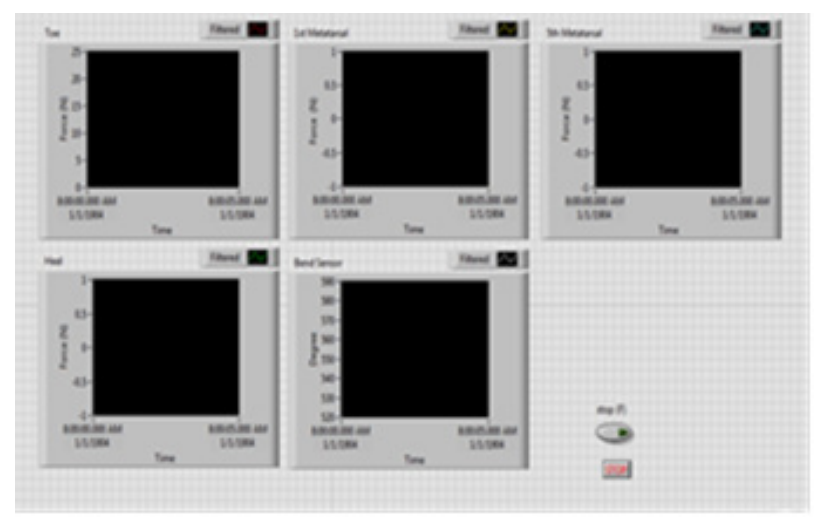

Figure 2. The measurement system graphical user. 
Figure 3 shows the complete instrumented insole system. Sensor is attached to the insole and wired into the signal conditioning circuit. The outputs of the signal conditioning circuit are connected to the DAQ device. DAQ connected to the laptop through USB connection.

\section{Protocol}

Subject need to wear proper shoes with the insole system inside their shoe. Subject then need to walk on a treadmill in a normal pace on. Walking speed must be in usual practice. It must be performed not too fast or slow. This simulation is to see the force distribution on the foot. Force at 1 st and 5th metatarsals, big toe, heel and plantar flexion angle in this activity is shown on the GUI and also recorded. The explanation of the gait parameters is shown in Table 1.

TABLE I.

THE GAIT PARAMETERS

\begin{tabular}{|l|l|}
\hline Type & Description \\
\hline Stance phase & Is a period when the foot in contact with grounding \\
\hline Heel strike & Is a period when the heel hit the grounding \\
\hline Mid-stance & $\begin{array}{l}\text { Is a period when toe, 1st and 5th metatarsal not in } \\
\text { contact with the grounding and the heel is rising. }\end{array}$ \\
\hline Terminal stance & Is a period when the toe, 1st and 5th metatarsal \\
\hline Pre swing (Toe-off) & $\begin{array}{l}\text { Is a period when 1st and 5th metatarsal in contact with } \\
\text { the grounding. Toe did not touch the ground. }\end{array}$ \\
\hline Swing phase & Is a period when foot not in contact with the grounding. \\
\hline Plantar flexion & Is a flexion during stance phase \\
\hline
\end{tabular}

\section{RESUlT AND DiSCUSSION}

Figure 4 shows the initial testing of the system during standing. The average value of force distribution on the foot is $20.9 \mathrm{~N}$ at heel, $9 \mathrm{~N}$ at the toe, $5.9 \mathrm{~N}$ at 5 th metatarsal, $0.5 \mathrm{~N}$ at $1 \mathrm{st}$ metatarsal region and $0.2^{\circ}$ angle at plantar flexion during 5 seconds of standing period. This distributions is varies on each test subject affected by subject body mass and sensor placement in this case.

Figure 5 shows the example of system GUI and sensors response during normal walking activities. The system has a capability in providing a real-time measurement of foot force and plantar flexion angle. However, in certain cases of gait analysis, a detail response of the signals also could be analyzed off-line. All measurement data could is recorded for further analysis.

Force transition at the foot starting from hind foot to forefoot. Initially, force exerted on the heel regions. When the heel is lifted, 5th metatarsal will contact with the treadmill so that force will be exerted on that region. Then 1st metatarsal and last region's contact with the treadmill is the big toe. Figure 6 shows there is the time interval between each region for 2 complete gait cycles. The time interval can be seen starting from the heel to the fifth metatarsal. The step time recorded in complete gait cycle is $1.63 \mathrm{~s}$. From heel strike to toe off, the time step starting from 0 to $0.1 \mathrm{~s}, 0.2 \mathrm{~s}, 0.25 \mathrm{~s}, 0.37 \mathrm{~s}$ accordingly. This result shows normal step time where smaller time intervals between phases of gait show that length of 1st metatarsal to the big toe is shorter. However, the step time measurement accuracy of this system is limited by DAQ device.

The plantar flexion angle during walking activities shown in Figure 7. The average angle calculated ranges from $8^{\circ}$ to $10^{\circ}$ which slightly different comparing meas-

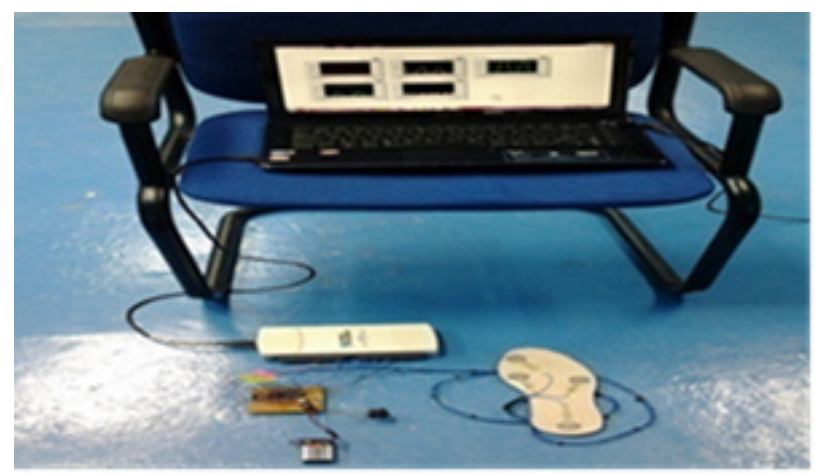

Figure 3. The instrumented insole system compromised hardware and software.

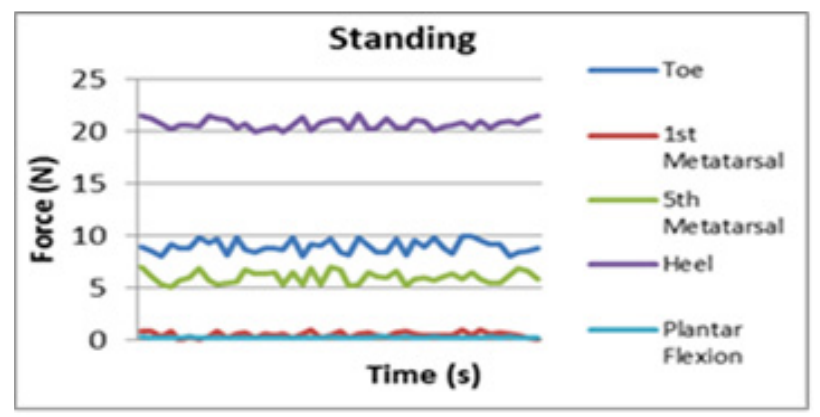

Figure 4. . Standing force distribution

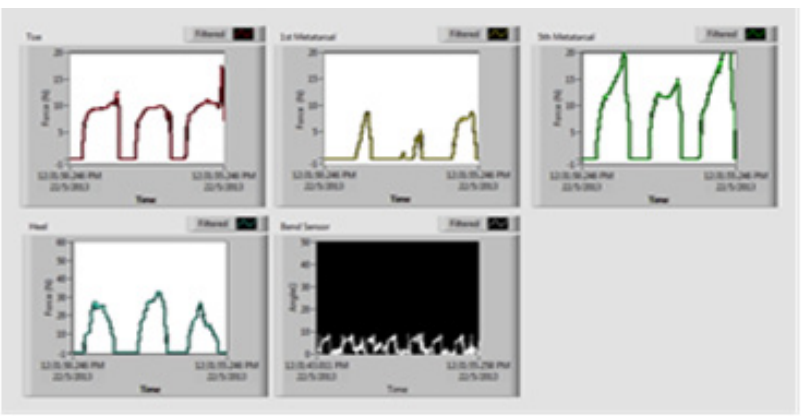

Figure 5. Sensors response to normal walking activities; (a) Toe, (b) 1st metatarsal, (c) 5th metatarsal, (d) Heel, (e) Plantar flexion.

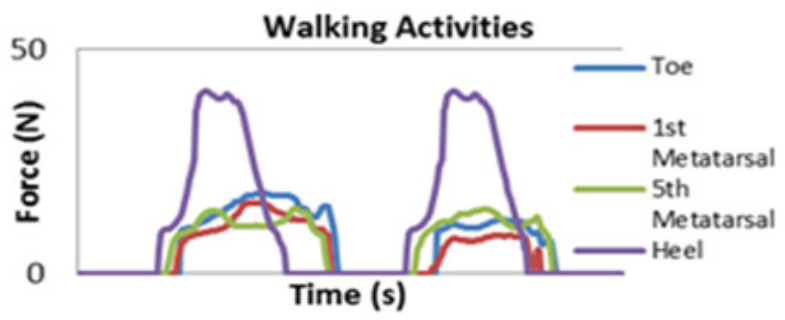

Figure 6. Force transition between each region of the right foot.

using Kinovea software which showing angle ranges from $18^{\circ}$ to $22^{\circ}$ during pre-swing (toe-off) position. However, the range of motion for plantar flexion is identified within $45^{\circ}$ to $50^{\circ}$ depending on the foot activities. While forces exerted at the toe, first and fifth metatarsals are lower than heel region. It shows that force distribution beneath the foot averages within range of $60 \mathrm{~N}$ to $80 \mathrm{~N}$. It has been identified that factors such as body weight and height is a will affect force distribution beneath the foot. The force of the foot is proportional to these factors as shown in Table II. 
TABLE II.

FORCE DISTRIBUTIONS ON THE RIGHT FOOT DURING WALKING

\begin{tabular}{lcccccc}
\hline Subjects & Weight $(\mathrm{kg})$ & Height $(\mathrm{cm})$ & \multicolumn{4}{c}{ Force $(\mathrm{N})$} \\
\hline & & & Heel & $5^{\text {th }} \mathrm{MH}$ & $1^{\text {st }} \mathrm{MH}$ & Toe \\
\hline 1 & 42 & 156 & 47 & 30 & 15 & 19 \\
\hline 2 & 48 & 154 & 55 & 33 & 21 & 30 \\
\hline 3 & 55 & 158 & 63 & 38 & 27 & 41 \\
\hline
\end{tabular}

Figure 8(a) shows the start of heel strike on the treadmill. The force is increasing due to the increasing body weight that heel needs to support. Maximum peak force at Figure 8(b) indicates the heel is starting to support the center of the body weight. Figure 8(c) shows the force is starting to decrease due to the time when the heel start to lift or not in contact with the treadmill. Zero value of force indicates that the heel is not touching or in contact with the treadmill. At this time, fifth metatarsal will take place then following with first metatarsal and big toe region.

\section{CONCLUSION}

The developed measurement system has been proved that it could be used to monitor and analysis gait depending on the foot pressure distributions. The sensing position is highly important to provide a valuable analysis use in many applications. However, in order to monitor or analyze details of force distribution on a foot, many points of sensing should be cover over the foot area. The foot pressure measurement is not an actual reading because FSR and FBS do not reflect to the actual pressure and bend flex angle due to the small active sensing area and also have a limited sensing range and sensors accuracy. Moreover, the force and flex angle (foot flexion) might occur only at one point and not represent the magnitude of load on that area. Therefore, the foot force analysis should cover the whole region which indicates the behavior and the individual actions of tendons, possibly ligaments.

The insole material also needs to be considered due to sensors attached to the surface or behind insole during force applied because of the insole soft foam surface which will absorb some of the foot force to the sensor. This could make the measurement of foot force inconsistent and also could lead to measurement error.

The insole must be adequately adapted to the foot to ensure the measurement is consistently reproducible and also less forces loss due to insole position in the shoe. Step time analysis accuracy of this system could be improved by applying a simultaneous data sampling to overcome multiplexing delay in the acquisition device.

Another way to improve the system is providing a wireless on the front-end of the system is proposed. A wired system is limited by walking activity which could only be performed on the treadmill and not on the freeway. This could limit the originality of gait. Moreover, the speed of movement is controlled by the treadmill, not by the subjects. Hence, the original gait pattern might be altered of this. On the other hand, the foot force has a deviation from the real conditions of force exhibited due to less effort of feet kinetics during walking because of the footstep cycle controlled by treadmill. Therefore, a wireless system which providing the uncontrolled environment and providing originality of gait is required to minimize error in the analysis.

It is also recommended that analysis of gait could be integrated together with many other systems, techniques and

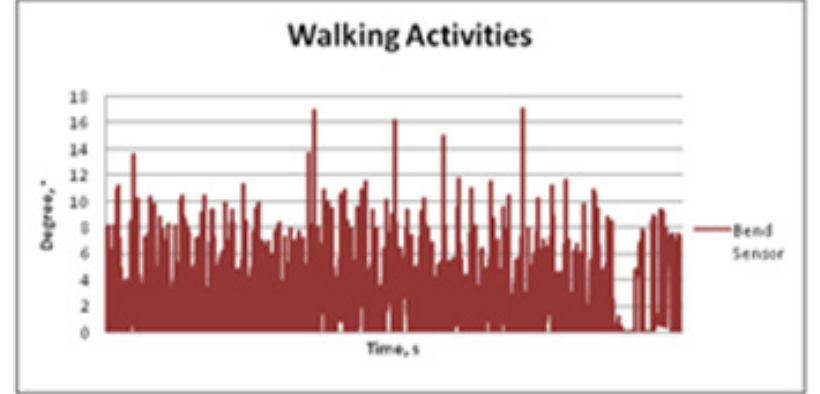

Figure 7. Plantar flexion angle during walking movement.

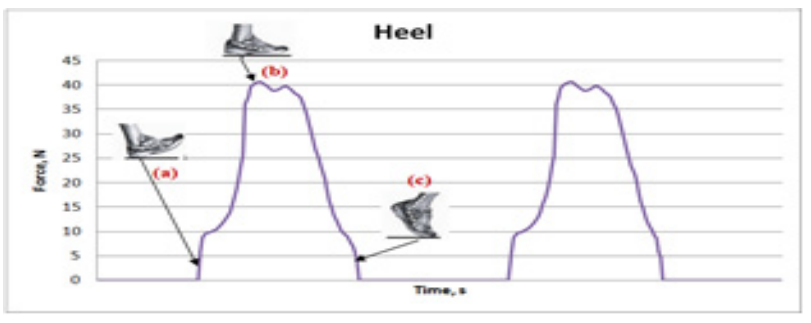

Figure 8. The heel force distribution

technology as resulted more parameters involve such as kinematic, kinetics and muscle signals which involved quantitate and qualitative result for greater accuracy analysis of gait conditions.

\section{ACKNOWLEDGMENT}

Author would like to thank to the staff of Biomedical Engineering, Universiti Malaysia Perlis for their help and support in this project.

\section{REFERENCES}

[1] Hausdorff JM: Gait variability: methods, modeling and meaning. J Neuroengineering Rehabil 2005;2:19. http://dx.doi.org/10.1186/ 1743-0003-2-19

[2] Mueller, M. J., Minor, S. D., Sahrmann, S. A., Schaaf, J. A., \& Strube, M. J. (1994). Differences in the gait characteristics of patients with diabetes and peripheral neuropathy compared with agematched controls. Physical therapy, 74(4), 299-308.

[3] Pedersen, S. W., Oberg, B., Larsson, L. E., \& Lindval, B. (1997). Gait analysis, isokinetic muscle strength measurement in patients with Parkinson's disease. Scandinavian journal of rehabilitation medicine, 29(2), 67-74.

[4] Boulgouris, N. V., Hatzinakos, D., \& Plataniotis, K. N. (2005). Gait recognition: a challenging signal processing technology for biometric identification. signal processing magazine, IEEE, 22(6), 78-90. http://dx.doi.org/10.1109/MSP.2005.1550191

[5] Lai, D. T., Begg, R. K., \& Palaniswami, M. (2009). Computational intelligence in gait research: a perspective on current applications and future challenges. Information Technology in Biomedicine, IEEE Transactions on, 13(5), 687-702. http://dx.doi.org/10.1109/TITB.2009.2022913

[6] Baker, R. (2005). Gait analysis methods in rehabilitation: Review. Journal of NeuroEngineering and Rehabilitation 2006, 3(4).

[7] S. Morris, J. Paradiso, Shoe-integrated sensor system for wireless gait analysis and real-time feedback, in: Proceedings of the 2nd Joint IEEE EMBS and BMES Conference, 2002, pp. 2468-246.

[8] Kirtley, C., Whittle, M. W., \& Jefferson, R. J. (1985). Influence of walking speed on gait parameters. Journal of Biomedical Engineering, 7(4), 282-288. http://dx.doi.org/10.1016/01415425(85)90055-X

[9] Von Schroeder, H. P., Coutts, R. D., Lyden, P. D., Billings, E., \& Nickel, V. L. (1995). Gait parameters following stroke: a practical assessment. Journal of rehabilitation research and development, $32,25-25$. 
PAPER

AN INSTRUMENTED INSOLE SYSTEM FOR GAIT MONITORING AND ANALYSIS

[10] Bamberg, S., Benbasat, A. Y., Scarborough, D. M., Krebs, D. E., \& Paradiso, J. A. (2008). Gait analysis using a shoe-integrated wireless sensor system. Information Technology in Biomedicine, IEEE Transactions on, 12(4), 413-423. http://dx.doi.org/10.1109/ TITB.2007.899493

[11] Kharb, A., Saini, V., Jain, Y. K., \& Dhiman, S. (2011). A REVIEW OF GAIT CYCLE AND ITS PARAMETERS. IJCEM International Journal of Computational Engineering \& Management, 13, 78-83.

[12] Cuccurullo, S. (Ed.). (2004). Physical medicine and rehabilitation board review. Demos Medical Publishing.

[13] Loudon, J., Bell, S., \& Johnston, J. M. (1998). The clinical orthopedic assessment guide. Human Kinetics.

[14] Davis III, R. B. (1988). Clinical gait analysis. Engineering in Medicine and Biology Magazine, IEEE, 7(3), 35-40. http://dx.doi.org/10.1109/51.7933

[15] Shaw, J. E., Van Schie, C. H., Carrington, A. L., Abbott, C. A., \& Boulton, A. J. (1998). An analysis of dynamic forces transmitted through the foot in diabetic neuropathy. Diabetes Care, 21(11), 1955-1959. http://dx.doi.org/10.2337/diacare.21.11.1955

[16] Simkin, A., \& Stokes, I. A. F. (1982). Characterisation of the dynamic vertical force distribution under the foot. Medical and Biological Engineering and Computing, 20(1), 12-18. http://dx.doi.org/10.1007/BF02441844
[17] Tao, W., Liu, T., Zheng, R., \& Feng, H. (2012). Gait analysis using wearable sensors. Sensors, 12(2), 2255-2283. http://dx.doi.org/10.3390/s120202255

[18] Morris, R. G., \& Lawson, S. E. M. (2009). A review and evaluation of available gait analysis technologies, and their potential for the measurement of impact transmission. Newcastle University, UK.

[19] Boukhenous, S. (2011). A Low Cost Three-Directional Force Sensor. International Journal on Smart Sensing and Intelligent Systems, 4(1), 21-34.

[20] Ledoux, W. R., \& Hillstrom, H. J. (2002). The distributed plantar vertical force of neutrally aligned and pes planus feet. Gait \& posture, 15(1), 1-9. http://dx.doi.org/10.1016/S0966-6362(01)00165-5

[21] Sparkfun.com.Spectra Symbol Flex Sensor, n.d. Web 10 April 2013.

\section{AUTHOR}

Anas M. N., is with the School of Mechatronic Engineering, Biomedical Electronic Program, Universiti Malaysia Perlis, 02600 Perlis, Malaysia (e-mail: anasnoor@ unimap.edu.my).

This work was supported by research grant (RAGS) number 9018-00043. Submitted 16 June 2014. Published as resubmitted by the author 25 October 2014. 